\title{
THE SET OF ALL ITERATES \\ IS NOWHERE DENSE IN $C([0,1],[0,1])$
}

\section{A. M. BLOKH}

\begin{abstract}
We prove that if a mixing map $f:[0,1] \rightarrow[0,1]$ belongs to the $C^{0}$-closure of the set of iterates and $f(0) \neq 0, f(1) \neq 1$ then $f$ is an iterate itself. Together with some one-dimensional techniques it implies that the set of all iterates is nowhere dense in $C([0,1],[0,1])$ giving the final answer to the question of A. Bruckner, P. Humke and M. Laczkovich.
\end{abstract}

\section{INTRODUCTION}

Let $C=C([0,1],[0,1])$ be the set of continuous self-mappings of the interval $[0,1]$ with $C^{0}$-topology. The generic properties of these maps were studied in a number of papers (see, e.g., [ABL, HL1-HL2, S1-S3]). One of the problems here, due to A. Bruckner, P. Humke and M. Laczkovich, concerns studying the generic properties of the sets $W(n)=\left\{f^{n}: f \in C\right\}$ and $W=$ $\bigcup_{n>2} W(n)$ in general and in particular the question of whether or not the set $W$ is nowhere dense in $C$.

The first results about the structure of the sets $W(n), W$ were obtained by P. Humke and M. Laczkovich who proved in [HL1-HL2] that $W(2)$ is not everywhere dense in $C$ and $W(k)$ is an analytic non-Borel subset of $C$. The next steps are due to K. Simon who proved the following

Theorem $\mathbf{S}$ [S1-S3]. 1. The set $W(2)$ is nowhere dense in $C$.

2. The set $W$ is of first category and $\bar{W} \neq C$.

Our aim is to study the problem in question using the method which is different from those of Humke-Laczkovich or Simon. First we prove Lemma 1 in which it is shown that if $h \in C$ and $h(0)=0$ or $h(1)=1$ then $h \in \bar{W}(2)$. Then we study properties of mixing interval maps (by mixing we always mean topologically mixing) and prove Theorem 1 which sums up these properties; to state it let us denote by $A$ the set of all interval maps with at least one fixed endpoint and by $M$ the set of all mixing maps (note that Lemma 1 makes the statement of Theorem 1 more natural).

Received by the editors August 8, 1990.

1991 Mathematics Subject Classification. Primary 26A18, 54H20, 58F03.

Key words and phrases. Iterates of maps, mixing maps, periodic points.

This paper was revised while I was visiting Wesleyan University; I would like to thank this institution for the kind hospitality. 
Theorem 1. $\bar{W} \cap M=(A \cap M) \cup(W \cap M)$; in other words, if a mixing map $f$ belongs to the closure of the set of iterates and $f(0) \neq 0, f(1) \neq 1$ then $f$ is an iterate itself.

We are going to apply Theorem 1 in order to find a dense open subset of $C$ containing no iterates. To this end we first develop in Lemma 8 some tools, which under certain conditions allow, roughly speaking, to "insert" a given interval map $g$ into another map $f$, i.e. to replace a fixed point of $f$ by an interval supporting $g$ and properly extend the new map. The idea of the rest is to find a special dense subset $B \subset C$ and then to "insert" properly chosen mixing maps (which are not iterates) instead of fixed points of any map $h \in B$. This way we get a dense set $D \subset C$ and the main step will be to prove that for any $\varphi \in D$ there exists an open set $U \ni \varphi$ containing no iterates; the techniques used in the proof involve some properties of periodic points of iterates of interval maps. Thus finally we obtain the following

Theorem 2. The set $W$ is nowhere dense in $C$.

The results of the paper were the subject of the author's talk during the Third Czechoslovak Summer Conference on Dynamical Systems near Bratislava in 1990.

\section{THE PROPERTIES OF MIXING MAPS}

We start with the foilowing

Lemma 1. (a) Let $h \in C$ and $h(0)=0$ or $h(1)=1$. Then $h \in \bar{W}(2)$.

(b) Let $f \in C, t=\min \{|f(0)-0|,|f(1)-1|\}$. Then $\operatorname{dist}(f, W(2)) \leq t$.

Proof. Let $A=\{f \in C: f(1)=1\}$ and $B=\{f \in A$ : there exists $a<1$ such that $f[0, a] \subset[0, a]$ and $f(x)<x$ for $x \in[a, 1)\}$. Let us show that $A=\bar{B}$. Indeed, let $f \in A$. Clearly for any open $U \ni f$ there exists $g \in A \cap U$ and $a<1$ such that $f x<x$ provided $x \in[a, 1)$. Let $\varepsilon<1-a$; construct a map $g_{\varepsilon}$ as follows: (1) $g_{\varepsilon}(x)=g(x)$ if $x \in[1-\varepsilon, 1]$ or $g(x)<1-\varepsilon$; (2) $g_{\varepsilon}(x)=1-\varepsilon$ otherwise. Then $g_{\varepsilon} \in B$ which proves the required since $g_{\varepsilon} \rightarrow g$ while $\varepsilon \rightarrow 0$ and on the other hand $g$ may be chosen arbitrary close to $f$.

Let us show that $B \subset \overline{W(2)}$. Indeed, let $h \in B$, i.e. there is $a<1$ such that $h[0, a] \subset[0, a], h x<x$ for $x \in(a, 1)$ and $h(1)=1$. Choose a point $b \in(a, 1)$ such that $c=h(b)>a$ and construct the map $g=g_{b}$ as follows:

(1) $g(0)=1, g(c)=b, g \mid[0, c]$ is linear;

(2) $g \mid[b, 1]$ is defined by the relation $g(x)=h\left(g^{-1} x\right)$ where $g^{-1} x \in[0, c]$;

(3) $g \mid[c, b]$ is linear.

Consider the map $g^{2}$. By the construction $g^{2}|[0, c]=h|[0, c]$. Furthermore, $g[c, b]=[h(c), b]$ which implies $g^{2}[c, b]=g[h(c), b] \subset[h(c), 1]$. Finally by the construction we have $g[b, 1]=h[0, c] \subset[0, c]$, so $g^{2}[b, 1] \subset$ $g[0, c] \subset[b, 1]$. Hence $g^{2}[c, 1] \subset[h(c), 1]$ and thus $\operatorname{dist}\left(g_{b}^{2}, h\right)=\operatorname{dist}\left(g^{2}, h\right)$ $\leq 1-h(c)=1-h^{2}(b) \rightarrow 0$ while $b \rightarrow 1$ which proves the statement (a) of the lemma.

(b) Let $t=|f(1)-1| \leq|f(0)-0|$. Let us consider a one-parameter family of maps $G=\left\{g_{\varepsilon}\right\}$ such that $g_{\varepsilon} x=f x$ for $0 \leq x \leq 1-\varepsilon, g_{\varepsilon}(1)=1$ and $g_{\varepsilon}$ is linear on the interval $[1-\varepsilon, 1]$. Clearly $\operatorname{dist}\left(g_{\varepsilon}, f\right) \rightarrow|f(1)-1|=t$ while $\varepsilon \rightarrow 0$. On the other hand $G \subset A$ and by the statement (a) we have $g_{\varepsilon} \in \overline{W(2)}(\forall \varepsilon)$. It completes the proof of Lemma 1 . 
Lemma 1 shows that for our purposes it is natural to consider the interval maps with nonfixed endpoints.

To proceed we need some well-known definitions. Namely, a family of continuous maps $g_{n}: X \rightarrow X$ of a metric space $(X, d)$ into itself is called equicontinuous if for any $t>0$ there is $r>0$ such that for any two points $x, y$ we have $d\left(g_{n} x, g_{n} y\right)<t$ provided that $d(x, y)<r$. Furthermore, a map $g: X \rightarrow X$ is called equicontinuous if the family of all its iterates $\left\{g^{n}\right\}_{n=0}^{\infty}$ is equicontinuous. The properties of equicontinuous interval maps are described in the following Lemmas 2 and 3.

Lemma 2. If a map of the interval $f: I \rightarrow I$ has the property $f^{2}=\mathrm{id}$ then $f$ is semiconjugate to either $\mathrm{id}:[0,1] \rightarrow[0,1]$ or $1-\mathrm{id}:[0,1] \rightarrow[0,1]$.

Proof. Left to the reader.

Lemma 3. Let $f \in C, I=[c, d]=\bigcap_{n \geq 0} f^{n}[0,1]$. Then $f$ is equicontinuous if and only if $f^{2}|I=\mathrm{id}| I$.

Proof. First of all let us observe that if there are points $a<b \in[0,1]$ such that $f a=a$ and $x<f x$ for all $x \in(a, b)$ then $f$ is not equicontinuous. Likewise, there are no points $a^{\prime}<b^{\prime} \in[0,1]$ such that $f b^{\prime}=b^{\prime}$ and $f y<y$ for $y \in\left[a^{\prime}, b^{\prime}\right)$ either. Moreover, the same conclusion concerns any iterate of $f$. This implies that if $f^{n} a=a, f^{n} b=b$ for some number $n$ and points $a, b$ then $f^{n} \mid[a, b]=\mathrm{id}$.

Now let $a=\min \{x: f x=x\}, b=\max \{x: f x=x\}$; then $f \mid[a, b]=\mathrm{id}$, all points from $[c, a)$ are mapped by $f$ to the right and all points from $(b, d]$ are mapped to the left. Since $f[c, d]=[c, d]$ this implies that $f[c, a] \supset[b, d]$ and $f[b, d] \supset[c, a]$. Hence there is a point $x \in[c, a]$ such that $f^{2} x=c$. If $x=c$ and $f^{2} c=c$ then by what was proven in the preceding paragraph we have $f^{2} \mid[c, a]=$ id and so $f^{2} \mid I=$ id. If $x \neq c$ then there is a point $e>x$ such that $f^{2} e=e$ and $f^{2}$ moves points from $[x, e)$ to the left which is impossible.

Let us show that if $f^{2} \mid I=$ id then $f$ is equicontinuous. Clearly it is enough to show that $f^{2}$ is equicontinuous; so we may assume that $f \mid I=\mathrm{id}$. We can also assume that $I$ is nondegenerate. Let $\varepsilon<\lambda(I)$ be given, $\delta<\varepsilon$ be such that $|f x-f y|<\varepsilon$ provided $|x-y|<\delta$ and $\delta^{\prime}<\delta$ be such that $|f x-f y|<\delta-\delta^{\prime}$ provided $|x-y|<\delta^{\prime}$. Then let us find $N$ such that $f^{N}[0,1]=\left[c_{N}, d_{N}\right] \subset\left[c-\delta^{\prime}, d+\delta^{\prime}\right]$ and $\delta^{\prime \prime}<\delta^{\prime}$ such that $\left|f^{i} z-f^{i} \zeta\right|<\delta^{\prime}$ for $0 \leq i \leq N$ provided $|z-\zeta|<\delta^{\prime \prime}$. We claim that if $|x-y|<\delta^{\prime \prime}$ then $\left|f^{i} x-f^{i} y\right|<\varepsilon$ for all $i$.

Indeed, by the choice of parameters we see that $\left|f^{i} x-f^{i} y\right|<\delta^{\prime}<\varepsilon$ for all $0 \leq i \leq N$ and at the same time $f^{N} x, f^{N} y \in f^{N}[0,1] \subset\left[c-\delta^{\prime}, d+\delta^{\prime}\right]$. So it is enough to show that $\left|f^{i} z-f^{i} z^{\prime}\right|<\varepsilon$ for any $i$ provided $z, z^{\prime} \in\left[c_{N}, d_{N}\right]$ and $\left|z-z^{\prime}\right|<\delta^{\prime}$. Indeed, otherwise let $j<\infty$ be the biggest number such that $\left|f^{i} z-f^{i} z^{\prime}\right| \leq \delta^{\prime}$ for all $0 \leq i \leq j$; then $\delta-\delta^{\prime}>\left|f^{j+1} z-f^{j+1} z^{\prime}\right|>\delta^{\prime}$. This implies that one of the points $f^{j+1} z, f^{j+1} z^{\prime}$, say, $f^{j+1} z$ belongs to $I$. If also $f^{j+1} z^{\prime} \in I$ then the required statement is proven since $f \mid I=\mathrm{id}$. Otherwise we may assume that $f^{j+1} z^{\prime} \in\left[c_{N}, c\right]$. Since $\delta-\delta^{\prime}>\left|f^{j+1} z-f^{j+1} z^{\prime}\right|$ then $\left|c_{N}-f^{j+1} z\right|<\delta<\varepsilon$, so as long as iterates of $z^{\prime}$ stay in $\left[c_{N}, c\right)$ the distance between them and $f^{j+1} z$ remain smaller than $\delta$. At the same time if $k$ is the least number such that $f^{k} z^{\prime} \notin\left[c_{n}, c\right)$ then by the choice of $\delta^{\prime}$ we have 
$\left|f^{k} z^{\prime}-c\right|<\varepsilon$ and so since $\varepsilon<\lambda(I)$ we have $f^{k} z^{\prime} \in I$ and $\left|f^{k} z^{\prime}-f^{j+1} z\right|<\varepsilon$. This completes the proof.

In the proof of Theorem 1 we need also the following

Lemma 4. Let $f:[0,1] \rightarrow[0,1]$ is mixing. Then the following holds:

(1) for any nondegenerate closed interval $K \subset(0,1)$ and any open interval I there exists $N$ such that $f^{n} I \supset K$ for all $n>N$;

(2) if $0<f(0), f(1)<1$ then there exists an interval $J \subset(0,1)$ such that $f J=[0,1]$

(3) if $f$ is piecewise-monotone then in fact for any $I$ there exists $N$ such that $f^{N} I=[0,1]$.

Proof. (1) This statement of the lemma follows immediately from the definition.

(2) Let $D$ be the set of those from points 0,1 which have no preimages in $(0,1)$ under iterations of $f$. Since $f$ is surjective then $D$ is $f^{-1}$-invariant. So the fact that $f(0), f(1) \in(0,1)$ implies that $D=\varnothing$ which in turn implies the statement (2).

(3) If $f$ is piecewise-monotone and the points 0 and 1 have preimages in $(0,1)$ under iterations of $f$ then the statement $(1)$ implies the required. Otherwise suppose that, say, 0 does not have the preimages in question; using the arguments and the notations from the preceding paragraph we see that either $0 \in D$ is $f$-fixed or $f(0)=1, f(1)=0$. So replacing if necessary $f$ by $f^{2}$ and taking into account that $f^{2}$ is also mixing we see that it remains to consider the following case: $f$ is mixing, $f(0)=0$ and 0 does not have preimages in $(0,1)$ under iterations of $f$. This easily implies that in fact 0 does not have preimages in $(0,1]$; indeed, if $f(1)=0$ then by surjectivity there exists a point $x \in(0,1)$ such that $f(x)=1$, and we have $f^{2}(x)=0$ which is a contradiction. Since $f$ is mixing and piecewise-monotone then 0 is a repeller and there is a small interval $(0, t]$ such that $f x>x$ for $x \in(0, t]$. Then $0 \notin f[t, 1]$ which together with the properties of $f \mid(0, t]$ implies that $f[t, 1] \cup[t, 1]$ is in fact an invariant proper subinterval of $[0,1]$. The existence of such an interval contradicts the fact that $f$ is mixing and thus completes the proof.

Recall that by $A$ we denote the set of all interval maps with at least one fixed endpoint, by $M$ the set of all mixing maps and by $W$ the set of all iterates. Now we are ready to prove the following

Theorem 1. $\bar{W} \cap M=(A \cap M) \cup(W \cap M)$; in other words, if a mixing map $f$ belongs to the closure of the set of iterates and $f(0) \neq 0, f(1) \neq 1$ then $f$ is an iterate itself.

Proof. Suppose that $f \in C$ is mixing, $f(0) \neq 0, f(1) \neq 1$ and there exists a sequence $\left\{\varphi_{n}\right\}, \varphi_{n} \in C$ and a sequence of numbers $\left\{p_{n}\right\}$ such that $\varphi_{n}^{p_{n}} \rightarrow$ $f, p_{n}>1$. It is enough to show that $f$ is an iterate, and we will do it step by step.

Step 1. $\mathscr{D}=\left\{\varphi_{n}^{i}\right\}_{n=1,0 \leq i \leq p_{n}}^{\infty}$ is an equicontinuous family of maps.

Suppose that $\mathscr{D}$ is not an equicontinuous family of maps. Then we may assume that there is a nondegenerate interval $I \subset(0,1)$, a point $a$, a sequence of intervals $\left\{J_{n}\right\}$ such that $J_{n} \rightarrow a$ and a sequence of integers $\left\{i_{n} \leq p_{n}\right\}$ such 
that $\varphi_{n}^{i_{n}}\left(J_{n}\right)=I$. Let $\varphi_{n}^{i_{n}+(l-1) p_{n}} J_{n}=K_{n}^{l}$; clearly $K_{n}^{l} \rightarrow f^{l-1} I$ while $n \rightarrow \infty$. On the other hand $\varphi_{n}^{l p_{n}} J_{n}=\varphi_{n}^{p_{n}-i_{n}} K_{n}^{l} \rightarrow f^{l} a$ while $n \rightarrow \infty$. By Lemma 4 $[1 / 3,2 / 3] \subset f^{l-1} I$ for large $l$; so for large $n$ and $l$ we have $K_{n}^{l} \cap K_{n}^{l+1} \neq \varnothing$ and thus $\varphi_{n}^{p_{n}-i_{n}} K_{n}^{l} \cap \varphi_{n}^{p_{n}-i_{n}} K_{n}^{l+1} \neq \varnothing$ which implies that $f^{l} a=f^{l+1} a$.

By the assumption 0,1 are not fixed points and so $f^{l} a \in(0,1)$. Then by Lemma 4 and since $f^{l} a$ is a fixed point we have that $f^{k} a=f^{l} a \in$ int $f^{k-1} I$ for large $k>l$. By the continuity arguments this implies that for sufficiently large $n$ we have $\varphi_{n}^{p_{n}-i_{n}} K_{n}^{k} \subset$ int $K_{n}^{k}$ where $\varphi_{n}^{p_{n}-i_{n}} K_{n}^{k} \rightarrow f^{k} a$ and $K_{n}^{k} \rightarrow f^{k-1} I$ while $n \rightarrow \infty$. Clearly there exists a nondegenerate closed interval $K \subset(0,1)$ such that $f^{k} a \in$ int $K \subset K_{n}^{k}$ and so $\varphi_{n}^{p_{n}-i_{n}} K \subset$ int $K$ for large $n$. On the other hand Lemma 4 implies that there is $m$ such that $\operatorname{int}\left(f^{m} K\right) \supset K$. Hence $\operatorname{int}\left(\varphi_{n}^{m p_{n}} K\right) \supset K$ for large $n$ and so $\operatorname{int}\left(\varphi_{n}^{m p_{n}\left(p_{n}-i_{n}\right)} K\right) \supset K$; at the same time we have just seen that $\varphi_{n}^{m p_{n}\left(p_{n}-i_{n}\right)} K \subset$ int $K$. Clearly this is a contradiction which completes Step 1.

Step 2. If $p_{n} \nrightarrow \infty$ then $f$ is an iterate.

We may assume that $p_{n}=p$ for any $n$. Since $\left\{\varphi_{n}\right\}$ is an equicontinuous family we may assume that $\varphi_{n} \rightarrow g \in C$. Obviously $f=g^{p}$.

Step 3. If there are infinitely many $p_{n}$ which are multiples of 3 then $f$ is an iterate.

Indeed, we may assume that all $p_{n}$ are multiples of 3 ; it is enough now to replace all $\varphi_{n}$ by $\psi_{n}=\varphi_{n}^{p_{n} / 3}$ and apply Step 2 to $\psi_{n}^{3} \rightarrow f$.

It remains to consider the case when $p_{n} \rightarrow \infty, p_{n}=3 q_{n}+r_{n}$ and $r_{n}=$ $2,4(\forall n)$. We may assume that $r_{n}=2(\forall n)$ (the other case can be treated similarly) and that $\varphi_{n} \rightarrow g$. Then $g$ is surjective. Indeed, otherwise if $[a, b]=$ $g[0,1] \varsubsetneqq[0,1]$ then there is an interval $[c, d]$ such that $[a, b] \varsubsetneqq[c, d] \subsetneq$ $[0,1]$ and for large $n$ we have $\varphi_{n}[0,1] \subset[c, d]$. So $\varphi_{n}^{p_{n}}[0,1] \subset[c, d]$ which is a contradiction since $\varphi_{n}^{p_{n}} \rightarrow f$ and $f$ is surjective. On the other hand by Step 1 and since $p_{n} \rightarrow \infty$ we see that $g$ is an equicontinuous map; thus by Lemma $2 g^{2}=$ id. By Step 1 the family of maps $\left\{\varphi_{n}^{i}\right\}_{0 \leq n, 0 \leq i \leq p_{n}}$ is equicontinuous. Therefore we may assume that $\varphi_{n}^{q_{n}} \rightarrow h$ which implies that $\varphi_{n}^{p_{n}}=\varphi_{n}^{3 q_{n}+2} \rightarrow h^{3} g^{2}=h^{3}=f$. This completes the proof.

Corollary 1 illustrates the way Theorem 1 may be applied. We need the following Proposition 1 concerning the estimates of the topological entropy $h(f)$ of a transitive interval map $f$ ([B1-B2] or [BC]); for the sake of completeness we will give here a short proof for a mixing case only.

Proposition 1 [B1-B2, BC]. Let $f:[0,1] \rightarrow[0,1]$ be mixing. Then $h(f) \geq$ $(\ln 2) / 2$. Moreover, if there is more than one $f$-fixed point then $h(f) \geq \ln 2($ in particular this is the case if one of the points 0,1 is fixed).

Proof. It is well known (see, e.g., [BGMY]) that if for a map $g:[0,1] \rightarrow[0,1]$ there exist two nondegenerate intervals $I, J$ with disjoint interiors such that $g I \cap g J \supset I \cup J$ then $h(g) \geq \ln 2$ (if such intervals exist we say that $f$ has a 2-horseshoe). We will use this property in the proof which is divided into steps.

Step 1. If there exist two f-fixed points then $h(f) \geq 2$.

Suppose that $0 \leq a<b \leq 1, f a=a, f b=b$ and $z<f z$ for $z \in(a, b)$. Let us prove that $a \in f[b, 1]$. Indeed, otherwise $I=[b, 1] \cup f[b, 1] \neq[0,1]$ 
is an $f$-invariant interval which contradicts the transitivity. Choose the smallest $c \in[b, 1]$ such that $f c=a$; then $b<c$. It is easy to see that there exists $d \in(a, c)$ such that $f d=c$; therefore, $f[a, d] \cap f[d, c] \supset[a, d] \cup[d, c]$ which means that the intervals $[a, d]$ and $d, c]$ have the aforementioned property and completes Step 1. Note that if, say, 0 is a fixed point of $f$ then the transitivity of $f$ implies that there is at least one more $f$-fixed point and so Step 1 may be applied in this case.

Step 2. If there exists a fixed point $t \in(0,1)$ such that $f y>y$ for any $y \in[0, t)$ and $f y<y$ for any $y \in(t, 1]$ then $h(f) \geq(\ln 2) / 2$.

By Step 1 and Lemma 4 it is enough to show that $f^{2}$ has more than one fixed point. Indeed, otherwise for any $y \in[0, t)$ we have $y<f y, y<f^{2} y$ and so because of the surjectivity of $f$ there exists a point $c \in(t, 1]$ such that $f c=0$. Similarly we can conclude that there exists a point $d \in[0, t)$ such that $f d=1$. This implies that there exists a point $a \in[0, t)$ such that $f a=c$ and so $f^{2} a=0$ which is a contradiction.

Corollary 1. Let $g$ be mixing and $h(g)<\ln 2$. Then $g \notin \bar{W}$.

Proof. Suppose that $g \in \bar{W}$. Then by Theorem 1 one of the points 0,1 is fixed or $g$ itself is an iterate. By Proposition 1 in the first case $h(g) \geq \ln 2$, so this possibility is ruled out. Assume that $g=f^{k}, k>1$. Let us show that in fact $f$ is mixing. Indeed, since $g$ is surjective then $f$ is surjective as well. Thus for any closed interval $K \subset(0,1)$ there is a closed interval $L \subset(0,1)$ such that $K \subset f^{i} L, 0 \leq i \leq k$. Let $I$ be an open interval. By Lemma 4 there exists $j$ is such that $g^{i} I \supset L$ for all $i \geq j$ and hence for all $r \geq k j$ we have $f^{r} I \subset K$. Clearly this implies that $f$ is mixing. By Proposition 1 we may conclude that $h(f) \geq(\ln 2) / 2$ and so $h(g) \geq \ln 2$ which is a contradiction.

\section{Properties OF PERIODIC POINTS OF ITERATES AND "INSERTION LEMMA"}

Let us first prove some lemmas concerning properties of periodic points of iterates.

Lemma 5. Let $f \in C, I$ be an interval and $n$ be the least integer such that $f^{n} I \subset I$. Suppose that $n>1, n$ is an odd integer and in fact $f^{n} I=I$. Then $f^{i} I \cap f^{j} I=\varnothing$ where $0 \leq j<i<n$.

Proof. Let $J \supset I$ be the connected component of the set $M=\bigcup_{r=0}^{n-1} f^{r} I$ and $m$ be the least positive integer such that $f^{m} I \cap J \neq \varnothing$. Then $f^{m} J=J, M=$ $\bigcup_{r=0}^{m-1} f^{r} J$ and $f^{i} J \cap f^{j} J=\varnothing$ for $0 \leq i<j<m$; it implies that $n$ is a multiple of $m$ so that $k=n / m$ is an integer. If $m=n$ there is nothing to prove. Let $m<n$ and consider $f^{m}: J \rightarrow J$. Then denoting $f^{m}$ by $g$ and assuming $J=[0,1]$ we have that $g \in C, I$ is an interval and $k$ is the least number such that $g^{k} I \subset I$; additionally it is known that $g^{k} I=I, k \geq 3$, is odd and $\bigcup_{i=0}^{k-1} g^{i} I=[0,1]$. Let us show that this is impossible.

Replacing $I$ by its iterate if necessary, we may assume that $0 \in I$. Clearly $g(0) \neq 0$ (otherwise $I \subset g I$ or $g I \subset I$ where both possibilities are excluded); similarly $g(1) \neq 1$. Let $b=\max \{x: g x=x\}$; then $I=g^{k} I \supset[0, b]$ and $g x<x$ for $x \in(b, 1]$. Denoting by $c$ the right endpoint of the interval $g I$ we may conclude that $g[0, c] \subset[0, c]$ since $g[0, b] \subset[0, c]$ and $g[b, c] \subset[0, c]$. 
Together with $\bigcup_{i=0}^{k-1} g^{i} I=[0,1]$ this implies that $c=1$. Similarly it is easy to see that $0 \in g(g I)$ and so $g^{2} I \subset I$ or $g^{2} I \supset I$; clearly this contradicts the assumption and completes the proof.

Let $P$ be a periodic orbit of a map $f$. In what follows we call an interval $\left[x^{\prime}, x^{\prime \prime}\right]$ basic (for the periodic orbit $P$ ) if $x^{\prime}, x^{\prime \prime} \in P$ and $\left(x^{\prime}, x^{\prime \prime}\right) \cap P=\varnothing$; if additionally it is known that $f$ moves points $x^{\prime}, x^{\prime \prime}$ in different directions then we call $\left[x^{\prime}, x^{\prime \prime}\right]$ expanding basic (for the periodic orbit $P$ ) interval.

Lemma 6. Let $f \in C, n$ be a prime integer and $x$ be an $f$-periodic point of period $n$. Then the following properties hold:

(1) if $[a, b]$ is an expanding basic for orb $x$ interval then $f^{j}[a, b] \supset$ orb $x$ for any $j \geq n$

(2) for any $y \neq z \in$ orb $x$ and $j \geq 2 n$ we have $f^{j}[y, z] \supset$ orb $x$;

(3) if $n$ is odd then for any f-fixed point $c$ and any $y \in \operatorname{orb} x$ we have $f^{2 n}[c, y] \supset$ orb $x$.

Proof. (1) Let $f^{i}[a, b] \cap$ orb $x=B_{i}$; then $B_{i} \subset B_{i+1}$ for any $i$. At the same time if $B_{i}=B_{i+1}$ then $B_{i}$ is an invariant subset of a periodic orbit orb $x$ which is possible only if $B_{i}=$ orb $x$. Hence for any $i$ if $B_{i} \neq$ orb $x$ then $B_{i} \subsetneq B_{i+1}$, which implies that $B_{n}=$ orb $x$ and so $f^{n}[a, b] \supset$ orb $x$.

(2) Let $k<n$ be such that $f^{k} y=z$. Since $n$ is prime then the family of pairs of points $\left\{f^{i} y, f^{j} z\right\}_{i=0}^{n-1}$ coincides with the family of pairs of points $\left\{f^{i k} y, f^{(i+1) k} y\right\}_{i=0}^{n-1}$. Let us divide the set orb $x$ into two parts $L=\{a \in$ orb $x: f a<a\}$ and $R=\{b \in$ orb $x: f b>b\}$. Then there exists $i$ such that $f^{i k} y$ and $f^{(i+1) k} y=f^{i k} z$ belong to $L$ and $R$; so if $i k \equiv m(\bmod n)$ then $f^{m} y$ and $f^{m} z$ belong to $L$ and $R$. Hence there is an expanding basic for orb $x$ interval $[a, b] \subset\left[f^{m} y, f^{m} z\right]$; since $f^{i}[a, b] \supset[a, b]$ for any $i$ then $f^{n}[y, z] \supset[a, b]$. Applying the statement (1) of the lemma we see finally that $f^{2 n}[y, z] \supset f^{n}[a, b] \supset$ orb $x$.

(3) Suppose there exists an interval $[c, z]$ with $z \in$ orb $x$ which contains an expanding basic interval; then one can find $m<n$ such that $f^{m} y=z$ and apply statement (1) which implies the required. If there are no such intervals then obviously the only expanding basic interval in orb $x$ is the interval $[a, b]$ where $a<c<b$ and $a, b$ are adjacent points in orb $x$. Since $n$ is odd the numbers of points from orb $x$ lying to the left and to the right of $c$ are different; suppose for the definiteness that the number of points lying to the left is bigger and denote the leftmost point in orb $x$ by $\zeta$. Let $l<n, f^{l} y=\zeta$. Then $f^{l+1}[c, y] \supset f[c, \zeta] \supset[a, b]$ which together with the statement (1) implies the required.

Let $\left\{A_{i}\right\}$ be a sequence of sets; denote by $\left\{A_{i}\right\}^{\prime}$ the set of all limit points of all sequences $\left\{a_{i}\right\}$ where $a_{i} \in A_{m(i)}$ and $m(i) \rightarrow \infty$.

Lemma 7. Let $\left\{n_{i}\right\}$ be a sequence of prime integers and $\varphi_{i}^{n_{i}} \rightarrow f$. Also let $\left\{p_{i}\right\}$ be a sequence of points such that $\varphi_{i}^{n_{i}}\left(p_{i}\right)=p_{i}$ for any $i$. Then one of the following possibilities holds:

(1) $\operatorname{diam}\left(\operatorname{orb}_{\varphi_{i}}\left(p_{i}\right)\right) \rightarrow 0$;

(2) choosing a subsequence one can assume that there exist numbers $N, t>$ 0 such that $1<n_{i}<N$ for any $i$ and for any cycle $\operatorname{orb}_{\varphi_{i}}\left(p_{i}\right)$ a distance between any two points from the cycle is greater than $t$. 
Moreover, if additionally all $n_{i}$ are odd then there exists a sequence of $\varphi_{i}$-fixed points $\left\{a_{i}\right\}$ such that $a_{i} \rightarrow a$ and also $p_{i} \rightarrow p$ then $f^{2}[a, p] \supset\left\{\operatorname{orb}_{\varphi_{i}}\left(p_{i}\right)\right\}^{\prime}$ so that if $p=a$ then $\left.\operatorname{orb}_{\varphi_{i}}\left(p_{i}\right)\right\}^{\prime}=\{a\}$.

Proof. If the second possibility does not hold then one can assume that there exists a sequence of pairs of points $x_{i}^{(1)}, x_{i}^{(2)} \in \operatorname{orb}_{\varphi_{i}}\left(p_{i}\right)$ such that $\mid x_{i}^{(1)}-$ $x_{i}^{(2)} \mid \rightarrow 0$ where $x_{i}^{(1)}=x_{i}^{(2)}$ if and only if the point $p_{i}$ is $\varphi_{i}$-fixed. Clearly we may assume that for sufficiently large $i$ the points $p_{i}$ are not $\varphi_{i}$-fixed. Hence by Lemma 6.(2) we have $\operatorname{orb}_{\varphi_{i}}\left(p_{i}\right) \subset \varphi_{i}^{2 n_{i}}\left[x_{i}^{(1)}, x_{i}^{(2)}\right]$. Since $\varphi_{i}^{n_{i}} \rightarrow f$ and $\left|x_{i}^{(1)}-x_{i}^{(2)}\right| \rightarrow 0$ this implies that $\operatorname{diam}\left(\operatorname{orb}_{\varphi_{i}}\left(p_{i}\right)\right) \rightarrow 0$ and so the first possibility holds.

Assume now that additionally all $n_{i}$ are odd, there exists a sequence of $\varphi_{i}$-fixed points $\left\{a_{i}\right\}$ such that $a_{i} \rightarrow a$ and also $p_{i} \rightarrow p$. Then by Lemma 6(3) $\operatorname{orb}_{\varphi_{i}}\left(p_{i}\right) \subset \varphi_{i}^{2 n_{i}}\left[a_{i}, p_{i}\right]$. Therefore taking the limits we have $f^{2}[a, p] \supset$ $\left\{\operatorname{orb}_{\varphi_{i}}\left(p_{i}\right)\right\}^{\prime}$, so that if $p=a$ then $\left\{\operatorname{orb}_{\varphi_{i}}\left(p_{i}\right)\right\}^{\prime}=\{a\}$.

The next lemma is quite different from those we have just proved. It provides techniques necessary for the proof of Theorem 2 and seems to be both useful and natural; roughly speaking, it shows that under certain conditions one can "insert" a map $g$ into a map $f$ so that the new map $h$ will contain an invariant interval supporting $g$ and will be monotonically semiconjugate to $f$. To formulate Lemma 8 we need a few definitions. Namely, a nondegenerate interval $I$ is called a flat spot (of a given map $f$ ) if $f I$ is degenerate; an interval of monotonicity of a piecewise-monotone map $f$ is called a lap.

Lemma 8 ("Insertion lemma"). Let $f \in C$ be a piecewise-monotone map without flat spots and $\mathscr{A}$ be a finite set of $f$-fixed points. Furthermore, let $\left\{g_{a}\right\}_{a \in \mathscr{A}}$ be the family of interval maps and for any $a \in \mathscr{A}$ one of the following possibilities holds:

(a) $g_{a}(0)=0, g_{a}(1)=1$ and $f$ does not reverse the orientation at $a$;

(b) $g_{a}(0)=1, g_{a}(1)=0$ and $f$ reverses the orientation at $a$;

(c) $g_{a}(0)=g_{a}(1)=0$ and $a$ is a local maximum of $f$;

(d) $g_{a}(0)=g_{a}(1)=1$ and $a$ is a local minimum of $f$.

Then in any neighborhood $U$ of $f$ in $C$ there exists a map $h$ and a nonstrictly increasing map $\varphi \in C$ with the following properties:

(1) $f \varphi=\varphi h$ (i.e. $\varphi$ semiconjugates $h$ to $f$ );

(2) $\varphi^{-1}(x)$ is a point if $x \notin \bigcup_{n \geq 0} f^{-n}(a)$ for any $a \in \mathscr{A}$ and a nondegenerate interval otherwise;

(3) if $\varphi^{-1}(x)$ is a nondegenerate interval and $x \notin \mathscr{A}$ then $h \mid \varphi^{-1}(x)$ is monotonically increasing, decreasing or has exactly one turning point depending on the corresponding behavior of $f$ in a small neighborhood of $x$;

(4) $\varphi^{-1}(a)$ is a nondegenerate $h$-invariant interval and $h \mid \varphi^{-1}(a)$ is conjugate to $g$ for any $a \in \mathscr{A}$;

(5) if $g$ is piecewise-monotone and has no flat spots then we may assume that $h$ is piecewise-monotone and has no flat spots either;

(6) if $\mathscr{A}$ is the set of all $f$-fixed points then all $h$-fixed points belong to the set $\varphi^{-1}(\mathscr{A})$. 
Proof. First of all observe that it is enough to prove the lemma in the case when $\mathscr{A}=\{a\}$ contains one point and then apply it step by step which will prove the general case since the set of fixed points in question is finite. So from now on let $\mathscr{A}=\{a\}$.

Let $n$ be the number of laps of $f$ and $b_{m}=\operatorname{card}\left\{f^{-m}(a)\right\}$; then $b_{i} \leq n^{i}$. Fix a number $N \gg n$. For any point $x \in[0,1]$ let $\nu(x)$ be 0 if $a \notin \operatorname{orb}_{f}(x)$ and $N^{-k-1}$ if $k$ is the smallest number such that $f^{k} x=a$; for instance, $\nu(a)=N^{-1}$. Let us define two functions $\eta(x)=\sum_{y: y<x, \nu(y) \neq 0} \nu(y)$ and $\beta(x)=\sum_{y: y \leq x, \nu(y) \neq 0} \nu(y)$. By the definition $\beta(x)-\eta(x)=\nu(x)$. It is easy to see also that $\eta(0)=0$ and $\beta(1)=\sum_{i}\left(b_{i}-1\right) N^{-i-1}<\frac{n}{N-n}$. Let us consider the interval $[0,1+\beta(1)]=I$ and construct the following function $J$ which establishes a correspondence between points in $[0,1]$ and closed (perhaps degenerate) subintervals of $I$. Namely, for any $x \in[0,1]$ let $J(x)=$ $[x+\eta(x), x+\beta(x)]=[c(x), d(x)]$; then $\lambda(J(x))=\nu(x)$. Moreover, by the construction both functions $c(x)$ and $d(x)$ are monotonically increasing and one can say that $J$ is also monotonically increasing since for any $x_{1}<x_{2}$ we have $J\left(x_{1}\right)<J\left(x_{2}\right)$.

We are ready to construct the map $h=h_{N}$; the construction depends on the steps already made (i.e. in the end on $N$ ). In fact we first construct the map $h^{\prime}: I \rightarrow I$ and then translate it onto the interval $[0,1]$ by the linear map. Now, for any point $y \in I$ let $F(y)=\{x: c(x) \leq y\}$. Then by the definition one can easily see that in fact the set $F(y)$ is an interval of the form $[0, \gamma(y)]$ where $\gamma(y)$ is a monotonically increasing continuous function of $y$ with $\gamma(0)=0, \gamma(1+\beta(1))=1$. Furthermore, $\gamma^{-1}(x)=J(x)$. Let us show that using the properties of the map $\gamma$ one can "lift" the map $f$ to the map $h^{\prime}: I \rightarrow I$ so that $h^{\prime}$ will be semiconjugate to $f$ by $\gamma$ and other properties from the statements (1)-(5) of the lemma will hold.

Indeed, let us define $h^{\prime}$ in the following way. First observe that by the definition if $y=J(x)$ is a point then $J(f(x))$ is a point as well; set $h^{\prime}(x)=$ $J(f(x))$. Suppose that $x \neq a$ and $J(x)$ is nondegenerate. By the construction this means that there exists the smallest number $0<k<\infty$ such that $f^{k} x=a$ and so by the construction $J(f x)$ is also nondegenerate. The definition of the map $h^{\prime}$ on $J(x)$ will depend on the behavior of $f$ at $x$. Namely, if $f$ is increasing at $x$ define $h^{\prime}$ on $J(x)=[c(x), d(x)]$ as a linear map determined by $h^{\prime}(c(x))=c(f(x))$ and $h^{\prime}(d(x))=d(f x)$. If $f$ is decreasing at $x$ define $h^{\prime}$ on $J(x)=[c(x), d(x)]$ as a linear map determined by $h^{\prime}(c(x))=d(f(x))$ and $h^{\prime}(d(x))=c(f x)$. Now suppose that $x$ is a local minimum of $f$. Then let $z$ be the middle point of $J(x)$ and define $h^{\prime}$ on $J(x)=[c(x), d(x)]$ as a linear on $[c(x), z],[z, d(x)]$ map determined by $h^{\prime}(c(x))=h^{\prime}(d(x))=d(f x)$ and $h^{\prime}(z)=c(x)$. Finally if $x$ is a local maximum of $f$ then let $z$ be the middle point of $J(x)$ and define $h^{\prime}$ on $J(x)=[c(x), d(x)]$ as a linear on $[c(x), z],[z, d(x)]$ map determined by $h^{\prime}(c(x))=h^{\prime}(d(x))=d(f x)$ and $h^{\prime}(z)=d(x)$. It remains to define $h^{\prime}$ on $J(a)$; we do it in such a way that it will be conjugate to $g$ by a strictly increasing map.

The construction immediately implies that $h^{\prime}$ has all the properties described in the statements (1)-(5) of the lemma. It is obvious for $h$ outside and inside $J(a)$; the continuity of $h$ at the endpoints of $J(a)$ follows from the connection between the properties of $g$ and the properties of the map $f$ at $a$ guaranteed 
by the conditions (a)-(d) of the lemma. Therefore if $h$ is the translation of $h^{\prime}$ on $[0,1]$ by the linear map then $h$ will have the same properties (a nonstrictly map $\varphi:[0,1] \rightarrow[0,1]$ which should semiconjugate $h$ and $f$ will correspond to the map $\gamma: I \rightarrow[0,1])$. By the construction it is easy to see that $I \rightarrow[0,1]$ and $\varphi \rightarrow$ id while $N \rightarrow \infty$; this implies that in fact $h \rightarrow f$ while $N \rightarrow \infty$. It remains to observe that if $\mathscr{A}$ is the set of all $f$-fixed points which was covered after the corresponding number of steps then the statement (6) holds too since we do not add new fixed points in the construction. This completes the proof.

\section{MAIN THEOREM}

In this section we prove the main theorem of the paper, namely Theorem 2. The main idea of the proof is to find a special dense subset $B \subset C$ and then to "insert" properly chosen mixing maps (which are not iterates) instead of fixed points of any map in $B$. The obtained dense set $D \subset C$ will have the property that for any $f \in D$ there exists an open set $U \ni f$ containing no iterates and establishing this property is in fact the major step of the proof.

We need the following definition. Let $\left\{A_{i}\right\}$ be a sequence of intervals and suppose that there is an interval $J$ such that for any intervals $I, L$ with $I \subset$ int $J, J \subset$ int $L$ and sufficiently big $i$ we have $I \subset A_{i} \subset L$; then we will say that the intervals $A_{i}$ converge to $J$ and denote it by $A_{i} \rightarrow J$.

Theorem 2. The set $W$ is nowhere dense in $C$.

Proof. First we construct maps $\left\{g_{i}\right\}_{i=1}^{4}$ with the properties which will allow to "insert" them into properly chosen interval maps and then to show that the resulting maps do not belong to the closure of the set of all iterates. Let $g:[1 / 3,2 / 3] \rightarrow[1 / 3,2 / 3]$ be a piecewise-monotone mixing map, $h(g)<$ $\ln 2,1 / 3<g(1 / 3), g(2 / 3)<2 / 3$; clearly such a map $g$ exists. All maps $g_{i}$ are defined in the similar way: they coincide with $g$ on $[1 / 3,2 / 3]$, they are linear on the intervals $[0,1 / 3]$ and $[2 / 3,1]$, but they act in different ways on points 0 and 1 . Namely, let the following hold:

$g_{1}(0)=0, g_{1}(1)=1 ; g_{2}(0)=1, g_{2}(1)=0 ; g_{3}(0)=g_{3}(1)=0 ; g_{4}(0)=$ $g_{4}(1)=1$.

For any $i$ and any interval $T$ such that $[1 / 3,2 / 3] \subset T \subset(0,1)$ there exists a number $m$ such that $g_{i}^{m} T=[1 / 3,2 / 3], 1 \leq i \leq 4$. Moreover, it is easy to see that there exists an interval $I$ such that $[1 / 3,2 / 3] \subset$ int $I$ and $g_{i} I=[1 / 3,2 / 3]$ for $1 \leq i \leq 4$. Finally note that by Lemma 4 there exists a unique $g$-fixed point in $[1 / 3,2 / 3]$ which does not coincide with $1 / 3,2 / 3$, so by the construction $g_{i}$ has no more than 3 fixed points.

We call a piecewise-monotone map $g$ expanding if there exists a number $\gamma>1$ such that for any interval $J$ the monotonicity (in the non-strict sense) of $f \mid J$ implies that $\lambda(f J) \geq \gamma \lambda(J)$.

Let $B$ be the set of piecewise-monotone and linear on each interval of monotonicity expanding maps outside $\bar{W}(2)$. By Theorem $\mathrm{S}$ the set $\bar{W}(2)$ is nowhere dense in $C$; together with some obvious arguments it easily implies that the set $B$ is dense in $C$. Moreover, any expanding map has finitely many fixed points. Now, let $f \in B$. Then by "insertion lemma" there exists a map $h \notin \overline{W(2)}$ obtained from $f$ by "inserting" the corresponding maps $g_{i}$ instead of all fixed 
points of $f$ and, furthermore, the map $h$ may be chosen arbitrarily close to $f$. We will prove that $h \notin \bar{W}$; clearly together with the fact that $B$ is dense this implies that $W$ indeed is nowhere dense in $C$. Suppose that there exists a sequence of maps $\left\{\varphi_{i}\right\}$ and a sequence of prime integers $\left\{n_{i}\right\}$ such that $\varphi_{i}^{n_{i}} \rightarrow h$ and show that it leads to the contradiction. Note that by the choice of $h$ all $n_{i}$ are odd.

Let $J=[a, b]$ be an $h$-fixed interval "inserted" instead of an $f$-fixed point. Then there is an increasing homeomorphism $\sigma: J \rightarrow[0,1]$ which conjugates $h \mid J$ and the corresponding $g_{i}$. Let $M=\sigma^{-1}[1 / 3,2 / 3], L=\sigma^{-1} I, K^{(i)}=$ $\bigcap_{m \geq 0} \varphi_{i}^{m n_{i}} L$ and show that $K^{(i)} \rightarrow M$. First observe that for any $K_{1} \subset$ int $M$ and sufficiently big $i$ we have $K_{1} \subset K^{(i)}$. Indeed, by the choice of $g$ and Lemma 4(2) there exists an interval $K^{\prime}$ such that $K_{1} \subset$ int $K^{\prime} \subset K^{\prime} \subset$ int $M$ and $h K^{\prime}=M$. Therefore for big $i$ we have $K^{\prime} \subset$ int $\varphi_{i}^{n_{i}} K^{\prime}$ which implies that in fact $K^{\prime} \subset \varphi_{i}^{m n_{i}} K^{\prime}$ for any $m$; so the fact that $K^{\prime} \subset L$ implies $K^{\prime} \subset K^{(i)}=$ $\bigcap_{m \geq 0} \varphi_{i}^{m n_{i}} L$ for big $i$. On the other hand by the choice of $L$ and properties of $I$ for any interval $K^{\prime \prime}$ such that $M \subset$ int $K^{\prime \prime}$ and big $i$ we have $\varphi_{i}^{n_{i}} L \subset K^{\prime \prime}$, so $K^{(i)} \subset K^{\prime \prime}$. Since both $K^{\prime}$ and $K^{\prime \prime}$ may be chosen arbitrary close to $M$ we have $K^{(i)} \rightarrow M$.

Obviously $\varphi_{i}^{n_{i}} K^{(i)}=K^{(i)}$. Let us show that either $\varphi_{i} K^{(i)}=K^{(i)}$ or $\varphi_{i}^{m} K^{(i)} \cap$ $\varphi_{i}^{k} K^{(i)}=\varnothing$ for $0 \leq m<k<n_{i}$. Indeed, by Lemma 5 it is enough to show that if $l$ is the least integer such that $\varphi_{i}^{l} K^{(i)} \subset K^{(l)}$ then $l=1$ or $l=n_{i}$. Suppose that $1<l<n_{i}$. Since $n_{i}$ is a prime integer then there exist integers $p, q$ such that $p n_{i}=q l+1$; it implies that $K^{(i)}=\varphi_{i}^{p n_{i}} K^{(i)}=\varphi_{i}^{q l+1} K^{(i)}$. On the other hand $\varphi_{i}^{q l} K^{(i)} \subset K^{(i)}$ and hence $K^{(i)}=\varphi_{i}\left(\varphi_{i}^{q l} K^{(i)}\right) \subset \varphi_{i} K^{(i)}$. The inclusion $K^{(i)} \varsubsetneqq \varphi_{i} K^{(i)}$ contradicts the relation $K^{(i)}=\varphi_{i}^{n_{i}} K^{(i)}$, so in fact $\varphi_{i} K^{(i)}=K^{(i)}$ which contradicts the properties of $l$.

Let us show that as a matter of fact the relation $\varphi_{i} K^{(i)}=K^{(i)}$ is excluded for sufficiently large $i$. Indeed, suppose that $\varphi_{i} K^{(i)}=K^{(i)}$ for arbitrary large $i$; taking a subsequence we may assume that this relation holds for all $i$. Since $K^{(i)} \rightarrow M$ then there exists a sequence of homeomorphisms $\zeta_{i}: K^{(i)} \rightarrow M$ such that $\max _{x \in K^{(i)}}\left|\zeta_{i}(x)-x\right| \underset{i \rightarrow \infty}{\longrightarrow} 0$. Define maps $\psi_{i}: M \rightarrow M$ as follows: $\psi_{i}=$ $\zeta_{i} \circ \varphi_{i} \circ \zeta_{i}^{-1}$. Then the properties of homeomorphisms $\zeta_{i}$ and the assumption that $\varphi_{i}^{n_{i}} \rightarrow h$ imply that $\psi_{i}^{n_{i}} \rightarrow h \mid M$ which is impossible because of the choice of $g$ and the construction of $h$. Therefore we may conclude that $K^{(i)} \rightarrow$ $M, \varphi_{i}^{n_{i}} K^{(i)}=K^{(i)}$ and $\varphi_{i}^{l} K^{(i)} \cap \varphi_{i}^{r} K^{(i)}=\varnothing\left(0 \leq l<r<n_{i}\right)$.

By the choice of $g$ it has a single fixed point which does not coincide with $1 / 3$ or $2 / 3$; hence $h \mid M$ has a single fixed point which does not belong to the boundary of $M$ and so by the construction $h \mid J$ has only one fixed point in its interior and at most two fixed points on its boundary. Denote the $h$-fixed point belonging to $M$ by $\alpha$; then $\alpha \in$ int $M$. Let $\alpha_{i} \in K^{(i)}$ be a $\varphi_{i}$-periodic point of period $n_{i}$. Then $\operatorname{orb}_{\varphi_{i}} \alpha_{i} \cap K^{(i)}=\left\{\alpha_{i}\right\}$. Moreover, the uniqueness of the $h$-fixed point $\alpha \in M$ implies that $\alpha_{i} \rightarrow \alpha$. Since in fact $\alpha \in$ int $M$ and at the same time $K^{(i)} \rightarrow M$ we have that $\operatorname{diam}\left(\operatorname{orb}_{\varphi_{i}} \alpha_{i}\right)>1 / 2 \cdot \operatorname{dist}(\alpha, \partial M)>0$ for large $i$; thus by Lemma 7 we may assume that $n_{i}=p$ for some odd prime integer $p$ and there exist $p$ distinct $h$-fixed points $z_{0}=\alpha, z_{1}, \ldots, z_{p-1}$ such that $\varphi_{i}^{l}\left(\alpha_{i}\right) \rightarrow z_{l}(0 \leq l<p)$ while $i \rightarrow \infty$. Moreover, by what has just been proven we see that $\left\{z_{l}\right\}_{l=0}^{p-1} \cap$ int $J=\left\{z_{0}\right\}$. Note that all arguments remain 
true for any "inserted" $h$-fixed interval, so we may assume that all conclusions hold for any such interval.

Now let us choose a sequence $\beta_{i}$ of $\varphi_{i}$-fixed points and suppose that $\beta_{i} \rightarrow \beta$; then $h(\beta)=\beta$ and by the properties of $h$ following from the "insertion lemma" there is an "inserted" interval $\widetilde{J}=[\tilde{a}, \tilde{b}]$ such that $\beta \in \tilde{J}$. On the other hand by what was shown there exist an odd prime integer $p$, a sequence $\left\{\tilde{\alpha}_{i}\right\}$ of $\varphi_{i}$-periodic points of period $p$ and $p$ distinct $h$-fixed points $\tilde{z}_{0} \in$ int $\widetilde{J}$, $\tilde{z}_{1}, \ldots, \tilde{z}_{p-1}$ such that $\varphi_{i}^{l}\left(\tilde{\alpha}_{i}\right) \rightarrow \tilde{z}_{l}(0 \leq l<p)$ while $i \rightarrow \infty$. Moreover, we may assume that $\left\{\tilde{z}_{l}\right\}_{l=0}^{p-1} \cap$ int $J=\left\{\tilde{z}_{0}\right\}$. Now if $\beta=\tilde{z}_{l}$ for some $l$ then by Lemma $7\left\{\operatorname{orb}_{\varphi_{i}} \tilde{\alpha}_{i}\right\}^{\prime}=\left\{\tilde{z}_{l}\right\}$ which is a contradiction. Hence $\beta \neq \tilde{z}_{0}$ and we may assume that, say, $h(\tilde{a})=\tilde{a}$ and $\beta=\tilde{a} \notin\left\{\tilde{z}_{l}\right\}_{l=0}^{p-1}$. Since $p \geq 3$ and at the same time $h \mid \tilde{J}$ has no more than 3 fixed points we conclude that $\left\{\tilde{z}_{l}\right\}_{l=0}^{p-1} \not \subset \tilde{J}$. On the other hand by Lemma $7\left\{\tilde{z}_{l}\right\}_{l=0}^{p-1}=\left\{\operatorname{orb}_{\varphi_{i}} \alpha_{i}\right\}^{\prime} \subset h^{2}\left[\tilde{a}, \tilde{z}_{0}\right] \subset \tilde{J}$ which is a contradiction completing the proof.

\section{ACKNOWLEDGMENTS}

I would like to thank the referee for useful remarks.

\section{REFERENCES}

[ABL] S. J. Agronsky, A. M. Bruckner, and M. Laczkovich, Dynamics of typical continuous functions, J. London Math. Soc. (2) 40 (1989), 227-243.

[BC] L. Block and E. Coven, Topological conjugacy and transitivity for a class of piecewise monotone maps of the interval, Trans. Amer. Math. Soc. 300 (1987), 297-306.

[BGMY] L. Block, J. Guckenheimer, M. Misiurewicz and L.-S. Young, Periodic points and topological entropy of one-dimensional maps, Lecture Notes in Math., vol. 819, Springer, 1980, pp. 18-34.

[B1] A. M. Blokh, On sensitive mappings of the interval, Russian Math. Surveys 37 (1982), 203-204.

[B2] _ The "spectral" decomposition for one-dimensional maps, Inst. for Math. Sci., SUNY at Stony Brook, Preprint \#1991/14, September 1991; Dynamics Reported (to appear).

[HL1] P. D. Humke and M. Laczkovich, Approximation of continuous functions by squares, Ergodic Theory Dynamical Systems 10 (1990), 361-366.

[HL2] - The Borel structure of iterates of continuous functions, Proc. Edinburgh Math. Soc. 32 (1989), 483-494.

[S1] K. Simon, Typical functions are not iterates, Acta Math. Hungar. 55 (1990), 133-134.

[S2] _ The set of second iterates is nowhere dense in C, Proc. Amer. Math. Soc. 111 (1991), 1141-1150.

[S3] - The iterates are not dense in C, Math. Pannon. 2 (1991), 71-76. 0128

Department of Mathematics, Wesleyan University Middletown, Connecticut 06459-

Current address: Department of Mathematics, University of Alabama at Birmingham, University Sta., Birmingham, Alabama 35294

E-mail address: ablokh@jordan.math.wesleyan.edu 